\title{
Current fluctuation in single-hole transport through a two-dimensional Si multidot
}

\author{
Ratno Nuryadi, Hiroya Ikeda, Yasuhiko Ishikawa, and Michiharu Tabe ${ }^{a)}$ \\ Research Institute of Electronics, Shizuoka University, 3-5-1 Johoku, Hamamatsu 432-8011, Japan
}

(Received 3 September 2004; accepted 26 January 2005; published online 22 March 2005)

\begin{abstract}
Single-hole transport in a two-dimensional Si multidot-channel field-effect transistor is studied. It is found that the single-hole-tunneling current fluctuates in the particular ranges of drain voltage and gate voltage. Such a phenomenon can be explained by a model that the hole transport through the percolation path is sensitively influenced and fluctuates with the time due to charging-discharging and polarity-switching of the dots adjacent to the percolation path. A Monte Carlo simulation using a parallel-double-dot circuit shows good agreement with the experimental characteristics. () 2005 American Institute of Physics. [DOI: 10.1063/1.1883705]
\end{abstract}

A two-dimensional (2D) Si quantum multidot structure has attracted interest because of its importance both in physics and device applications. In applying the Si multidot structure to new functional devices such as quantum cellular automata $(\mathrm{QCA})^{1,2}$ and photoimaging devices, ${ }^{3}$ it is necessary to study the electrical transport of the 2D Si dot array. Previously, it has been reported that, if the size of dots in the array is random, one current percolation path is formed at first due to the electrostatic potential variations in the low voltage region. ${ }^{4-6}$ The path is expected to be a onedimensional (1D) dot array. Although the transport characteristics are mainly determined by the circuit parameters of the path itself, the charging effect of the dots adjacent to the path (referred to as "adjacent dots") cannot be neglected due to the Coulomb interaction between the adjacent dots and the dots in the path.

Recently, Emiroglu et al. reported the characteristics of a $\mathrm{Si}$ single-electron transistor composed of a $\mathrm{Si}$ single dot, where adjacent $\mathrm{Si}$ double dots are capacitively coupled to the single dot (Coulomb island). ${ }^{7}$ They demonstrated a suppression of current and a modulation of the Coulomb blockade (CB) peak height due to single-electron polarization of the adjacent double dots. In the 2D dot system, the multidots surround the percolation path, so that the adjacent dot effect becomes more crucial. In this letter, we present results on the single-hole-tunneling transport in a 2D Si multidot-channel field-effect transistor (FET). It will be shown that the charging-discharging and polarity-switching in the adjacent dots induce the current fluctuation in the particular ranges of drain voltage and gate voltage.

The device used in this experiment is the 2D Si multidot FET fabricated on a silicon-on-insulator (SOI) substrate, as schematically shown in Fig. 1(a). The channel width and length are about 0.5 and $0.8 \mu \mathrm{m}$, respectively. In the channel, the Si dots are connected to each other via an ultrathin (about $5 \mathrm{~nm}$ ) Si layer, which works as a tunnel barrier due to the quantum size effect. The 2D multidots are formed by the nanometer-scale local oxidation of $\mathrm{Si}$ (nano-LOCOS) process, which has mainly two steps. ${ }^{6,8}$ First, the thin SOI $[18 \mathrm{~nm}$-thick top $\mathrm{Si}(100) / 90 \mathrm{~nm}$ thick buried $\left.\mathrm{SiO}_{2} / \mathrm{n}^{+}-\mathrm{Si}(100)\right]$ surface was nitrided in a vacuum to natu-

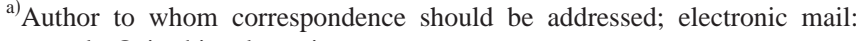
romtabe@rie.shizuoka.ac.jp
}

rally form ultrasmall $\mathrm{SiN}$ islands. Second, the surface was oxidized by a conventional furnace oxidation. Since the $\mathrm{SiN}$ islands work as the oxidation masks, $\mathrm{Si}$ multidots can be obtained below the SiN masks. The lateral size, height and density of the fabricated Si dots are about $20 \mathrm{~nm}, 3 \mathrm{~nm}$, and $2 \times 10^{11} \mathrm{~cm}^{-2}$, respectively, although the size and spacing of dots are distributed due to the naturally formed $\mathrm{SiN}$ masks. The $\mathrm{n}^{+}-\mathrm{Si}$ substrate works as the backgate and no top gate is prepared. The channel-source and channel-drain contacts are the Al Schottky contacts. Thus, the carrier polarity in the channel can be selected by the polarity of backgate voltage $\left(V_{b g}\right)$. For the positive $V_{b g}$, electrons are injected into the channel and the FET acts as a single-electron device, while, for the negative $V_{b g}$, holes are induced and the FET acts as a single-hole device. ${ }^{6}$

Figure 1(b) shows the drain current $\left(I_{d}\right)$ versus $V_{b g}$ characteristics for holes at $15 \mathrm{~K}$ with different drain voltages $\left(V_{d}\right)$. The current oscillations due to the CB effect are observed. As reported in our previous works, ${ }^{6,9}$ the CB oscillations are ascribed to the formation of the current percolation path in the 2D multidot channel, and a few dots having the highest-resistance tunnel junctions in the path should dominate the carrier transport. It can be seen in Fig. 1(b) that the spacing between the current peaks in the $\mathrm{CB}$ oscillations is

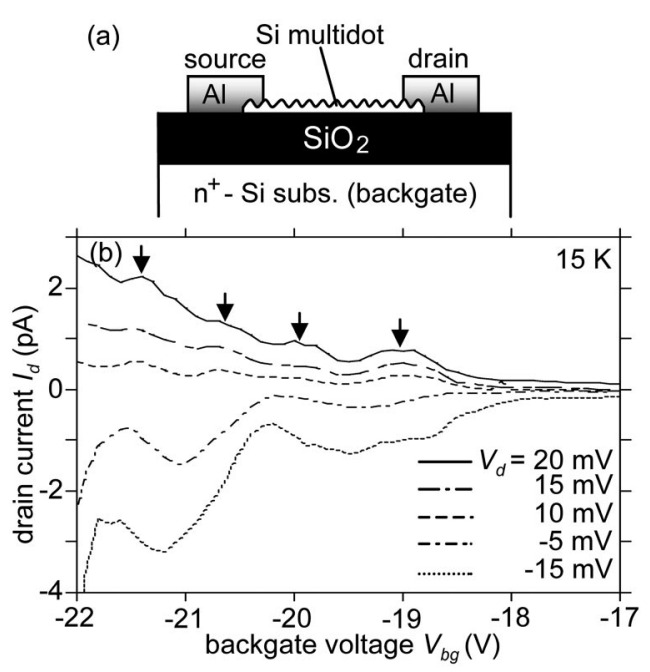

FIG. 1. (a) Schematic cross section of the 2D Si multidot-channel FET and (b) $I_{d}-V_{b g}$ characteristics for holes at $15 \mathrm{~K}$. 

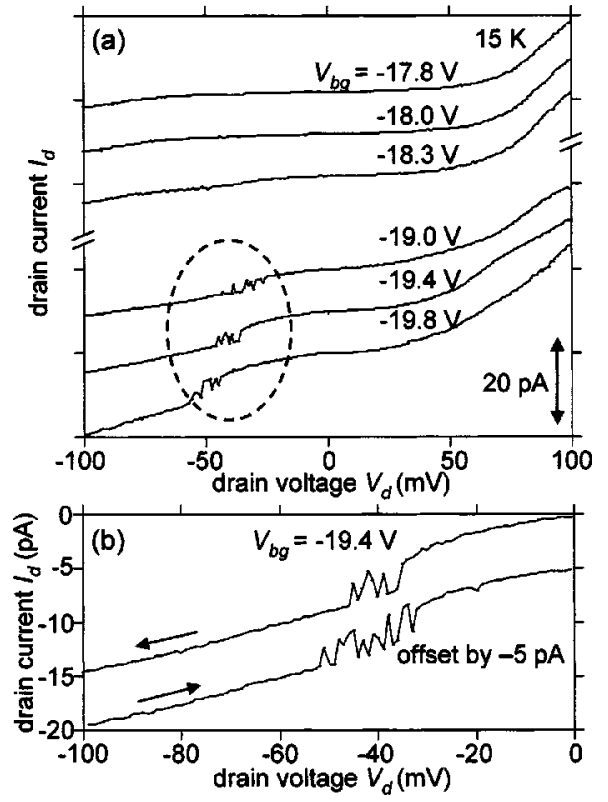

FIG. 2. (a) $I_{d}-V_{d}$ characteristics at $15 \mathrm{~K}$ for various $V_{b g}$ and (b) $I_{d}-V_{d}$ curves for upward and downward $V_{d}$ ramping with a sweep rate of $0.5 \mathrm{mV} / \mathrm{s}$. Arrows in Fig. 2(b) indicate the $V_{d}$ ramping directions.

not constant, indicating that a few dots are responsible for the observed current oscillations. ${ }^{10,11}$ Furthermore, it should be noted that the current-peak spacing for the positive $V_{d}$ is quite different from that for the negative $V_{d}$. This strongly suggests that the current path is changed by the polarity of $V_{d}$. This conclusion is consistent with the results in Fig. 2(a), shown next, where the drain current fluctuation is observed only for the negative $V_{d}$.

Figure 2(a) shows $I_{d}-V_{d}$ characteristics at $15 \mathrm{~K}$ for various $V_{b g}$. It is found that the $I_{d}-V_{d}$ curves with the current fluctuation are clearly detected in the negative $V_{d}$ region (indicated by a dashed circle) for the negative $V_{b g}$ of -19.0 to $-19.8 \mathrm{~V}$. Such a current fluctuation is not observed for the other $V_{b g}$ region, for example, $-17.8 \mathrm{~V}<V_{b g}$ $<-18.3 \mathrm{~V}$. It is noted that the meaning of the current fluctuation here is that the current fluctuates with the time, as shown below [Figs. 2(b) and 5(b)]. Figure 2(b) shows the $I_{d}-V_{d}$ curves for the upward and downward $V_{d}$ ramping with a sweep rate of $0.5 \mathrm{mV} / \mathrm{s}$. The fine structures of the drain current are different between two curves, indicating that the drain current fluctuates with the time. We could not observe such a fluctuation for FETs without the Si multidot structure (that is, flat-channel FETs). Therefore, the observed current fluctuation is likely to originate from the charging effect of the dots.

Taking into account the charging effect of the dots, we propose a parallel-double-dot model to explain the current fluctuation. Figures 3(a) and 3(b) show schematic diagrams of the parallel-double-dot structure and its simplified equivalent circuit, respectively. Since the $I_{d}-V_{b g}$ curves in Fig. 1(b) indicated that a few dots dominate the carrier transport, it is likely that the current percolation path is represented by a double-dot structure (dots 1 and 2). The dots 3 and 4 represent the dots adjacent to the path, whose charging states influence the tunneling transport. Charging of the adjacent dots occurs by the charge tunneling from the percolation path through the tunnel junction $\left(C_{13}\right.$ or $\left.C_{24}\right)$. Each of the dots is connected to a voltage source $\left(V_{g}\right)$ through a gate capacitor

Downloaded $10 \mathrm{Jul} 2008$ to $133.70^{\circ} .80 .50$. Redistribution subject to (a)

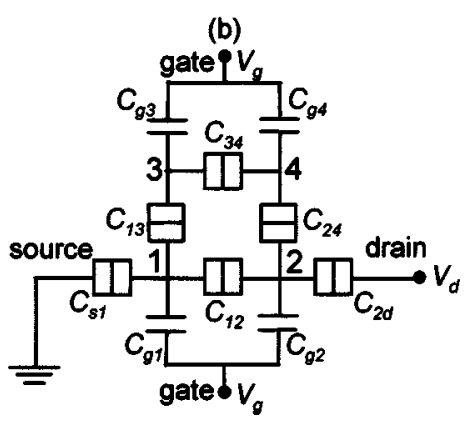

FIG. 3. (a) Schematic diagram of a parallel-double-dot structure and (b) its simplified equivalent circuit.

$\left(C_{g 1}, C_{g 2}, C_{g 3}\right.$ or $\left.C_{g 4}\right)$ representing the buried $\mathrm{SiO}_{2}$. We calculated the single-hole-tunneling characteristics of the circuit at $0 \mathrm{~K}$ using a Monte Carlo method. The calculation procedure is the same as in our previous works. ${ }^{3,12,13}$

Figure 4(a) shows a typical calculated $I_{d}$ contour plot in the $V_{d}-V_{g}$ plane, where we set the circuit parameters of $C_{s 1}=C_{12}=C_{2 d}=C_{13}=C_{24}=C_{34}=5 \mathrm{aF}, \quad C_{g 1}=C_{g 2}=C_{g 3}=C_{g 4}$ $=0.07 \mathrm{aF}, \quad R_{s 1}=R_{12}=R_{2 d}=100 \mathrm{k} \Omega, \quad$ and $\quad R_{13}=R_{24}=R_{34}$ $=100 \mathrm{M} \Omega$. In this figure, the brighter region represents the lower current. A strong suppression of the current around $V_{d}$ of $0 \mathrm{~V}$ is observed due to $\mathrm{CB}$. Furthermore, the noisy pattern, which will be found to reflect the current fluctuation with the time, is seen in the region $\mathrm{P} 1$, while the noisy pattern is absent in the region $\mathrm{Q} 1$. The $I_{d}-V_{d}$ characteristics drawn from the contour plot are shown in Fig. 5(a) for the P1 $\left(V_{g}=-0.6,-0.7\right.$, and $\left.-0.8 \mathrm{~V}\right)$ and Q1 $\left(V_{g}=0,-0.1\right.$, and $-0.2 \mathrm{~V})$ regions. These calculated results qualitatively agree with the experimental results in Fig. 2.

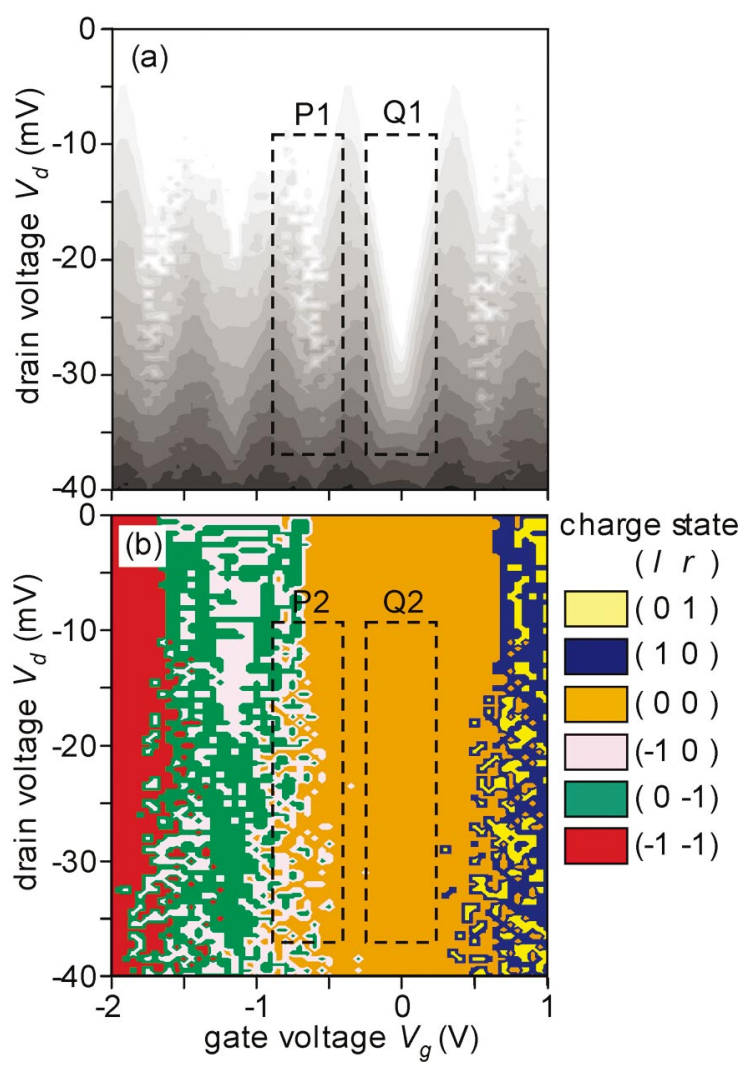

FIG. 4. (Color) (a) Calculated $I_{d}$ contour plot in the $V_{d}-V_{g}$ plane, and (b) a snapshot of the charge state $(l r)$ in the adjacent dots 3 and $4 . l$ and $r$ are the numbers of electrons in the dots 3 and 4 , respectively. 


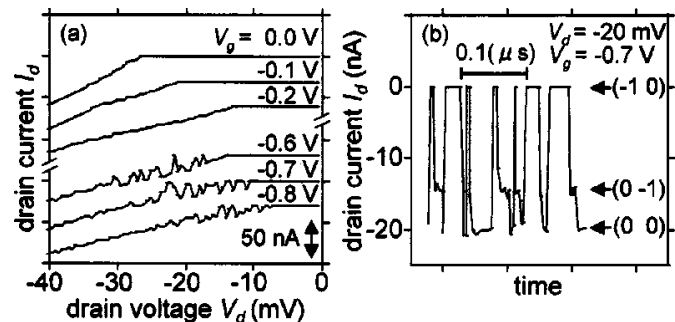

FIG. 5. (a) Calculated $I_{d}-V_{d}$ characteristics for $V_{g}=0,-0.1,-0.2,-0.6$, -0.7 , and $-0.8 \mathrm{~V}$; (b) calculated time-dependence of $I_{d}$ for $V_{d}=-20 \mathrm{mV}$ and $V_{g}=-0.7 \mathrm{~V}$.

In order to understand the origin of the noisy pattern in Fig. 4(a), a snapshot of charge state $(l r)$ in the adjacent dots is shown in Fig. 4(b), where $l$ and $r$ are the numbers of electrons in the dots 3 and 4, respectively. Corresponding to the noisy pattern in the region P1 of Fig. 4(a), the change in the charge state is seen in the region P2 of Fig. 4(b), while such a change is absent in the region Q2. Therefore, the current fluctuation in the $I_{d}-V_{d}$ curve is due to the change in the charge state $(l r)$. It is important that such a charge-state change randomly occurs with the time. Figure 5(b) shows the calculated time-dependence of $I_{d}$ for $V_{d}=-20 \mathrm{mV}$ and $V_{g}=-0.7 \mathrm{~V}$. The time dependence shows the random-telegraph-signal ${ }^{14,15}$ with three digital current levels, $0 \mathrm{nA}, \sim-15 \mathrm{nA}$ and $\sim-20 \mathrm{nA}$, corresponding to the charge states $\left(\begin{array}{ll}-1 & 0\end{array}\right),\left(\begin{array}{ll}0 & -1\end{array}\right)$, and $(00)$, respectively.

From Figs. 4(b) and 5(b), there are two types of the changes in the charge states. The first one is the charge tunneling from (to) the percolation path to (from) the adjacent dots through the junction $C_{13}$ or $C_{24}$. Such charging and discharging of the adjacent dots cause time-dependent potential fluctuation for the dot in the percolation path, leading to the current fluctuation. The second one is the change in the charge polarization of the adjacent dots due to single-charge switching from one of the double dots to the other dot through the junction $C_{34}$, for instance, between $(-10)$ and $(0-1)$ or between $\left(\begin{array}{ll}1 & 0\end{array}\right)$ and $(01)$. Such a polarity-switching in the adjacent double dots is crucial, because the paralleldouble-dot is the basic component of QCA. ${ }^{16,17}$

In conclusion, the current fluctuation in single-holetunneling characteristics of the 2D Si multidot-channel FET was observed in the particular ranges of drain voltage and gate voltage. This phenomenon is attributed to the timedependent charging-discharging and polarity-switching of the dots adjacent to the current percolation path.

We would like to thank Mr. T. Mizuno for his technical support in the experiment. This work is financially supported by a Grant-in-Aid for Scientific Research from JSPS.

${ }^{1}$ C. S. Lent, P. D. Tougaw, W. Porod, and G. H. Berstein, Nanotechnology 4, 49 (1993).

${ }^{2}$ C. S. Lent and P. D. Tougaw, Proc. IEEE 85, 541 (1997).

${ }^{3}$ M. Tabe, Y. Terao, R. Nuryadi, Y. Ishikawa, N. Asahi, and Y. Amemiya, Jpn. J. Appl. Phys., Part 1 38, 593 (1999).

${ }^{4}$ A. A. Middleton and N. S. Wingreen, Phys. Rev. Lett. 71, 3198 (1993).

${ }^{5}$ K. Uchida, J. Koga, R. Ohba, S. Takagi, and A. Toriumi, J. Appl. Phys. 90, 3551 (2001).

${ }^{6} \mathrm{R}$. Nuryadi, H. Ikeda, Y. Ishikawa, and M. Tabe, IEEE Trans. Nanotechnol. 2, 231 (2003).

${ }^{7}$ E. G. Emiroglu, D. G. Hasko, and D. A. Williams, Appl. Phys. Lett. 83, 3942 (2003).

${ }^{8}$ M. Tabe, M. Kumezawa, T. Yamamoto, S. Makita, T. Yamaguchi, and Y. Ishikawa, Appl. Surf. Sci. 142, 553 (1999).

${ }^{9}$ H. Ikeda, R. Nuryadi, Y. Ishikawa, and M. Tabe, Jpn. J. Appl. Phys., Part 2 43, L759 (2004).

${ }^{10}$ H. Ishikuro, T. Fujii, T. Saraya, G. Hashiguchi, T. Hiramoto, and T. Ikoma, Appl. Phys. Lett. 68, 3585 (1996).

${ }^{11}$ R. A. Smith and H. Ahmed, J. Appl. Phys. 81, 2699 (1997).

${ }^{12}$ M. Tabe, Y. Terao, N. Asahi, and Y. Amemiya, IEICE Trans. Electron. E81-C, 36 (1998).

${ }^{13}$ M. Tabe, N. Asahi, Y. Amemiya, and Y. Terao, Jpn. J. Appl. Phys., Part 1 36, 4176 (1999).

${ }^{14}$ M. Bollu, F. Koch, A. Madenach, and J. Scholz, Appl. Surf. Sci. 30, 142 (1987).

${ }^{15}$ A. Neugroschell, C. T. Sah, and M. S. Carroll, Appl. Phys. Lett. 66, 2879 (1995).

${ }^{16}$ M. Gattobigio, G. Iannaccone, and M. Macucci, Phys. Rev. B 65, 115337 (2003).

${ }^{17}$ C. Single, F. E. Prins, and D. P. Kern, Appl. Phys. Lett. 78, 1421 (2001). 